\title{
Group Theoretic Proof of Infinitude of Primes
}

\author{
Nazrul Haque
}

\begin{abstract}
There are numerous proofs on infinitude of primes using various tools. Starting from Euclid[1] to the analytical proofs given by Euler[2] and Paul Erdos[3]. The attractive proofs like one line proof by Northshield[4] and topological proof by Furstenberg[5] also fascinated the readers. We give here another proof of infinitude of primes using group theoretic argument.
\end{abstract}

Lemma. If there are finitely many primes namely $p_{1}, p_{2}, p_{3}, \ldots, p_{r}$, and $n=p_{1} . p_{2} . p_{3} \ldots . p_{r-1}$, also $n-1=p_{1} \cdot p_{2} \cdot p_{3} \ldots p_{r-1}-1$ then $|U(n)|=2, \forall n>2$ where, the group of units $U(n)$ is the set of numbers less than $n$ and relatively prime to $n$ under the operation multiplication modulo $n$ and $n \in \mathbb{N}$.

Proof. Assume that, $|U(n)|>2$.

Then there exists at least one $k \in U(n)$ other than $n-1$ such that $k$ is not a new prime.

Since, $\operatorname{gcd}(k, n)=1 \Longrightarrow k \neq \prod_{i=1}^{r} p_{i}{ }^{m_{i}},\left(m_{i} \in \mathbb{Z}^{+}\right)$.

Otherwise, $\operatorname{gcd}(k, n) \neq 1 \Longrightarrow k \notin U(n)$

It's only possible if $k=n-1$ or $k$ is a new prime. Both of these cases contradicts our assumption.

Since, $k$ is arbitrary there is no such $k \in U(n) \Longrightarrow|U(n)|=2$.

Theorem. There are infinitely many primes.

Proof. From the preceding section, it's clear that, $|U(n)|=2$, if the number of primes are finite.

It's known that, there are infinitely many $U(n)$ groups where the number of non-identity elements in $U(n)$ that satisfy the equation,

$$
x^{4}=1 \text {. }
$$

is a multiple of 4 i.e. $|U(n)|=4 s+t$, for infinitely many $n$ where $s$ and $t$ are arbitrary constants.

But, here in this case, $\forall n,|U(n)|=2$ i.e. there is only one non-identity element that satisfies the equation. (contradiction)

Hence, it is proved that, primes can not be finite. So, there are infinite number of primes. 


\section{Summary:}

The upper proof looks quite similar in flavor with Euclid's proof. But, in that proof the basic idea was - greatest common divisor of two consecutive natural numbers is one. Thus, there exists at least one more distinct prime.

We have constructed our proof in such a way that, it does not required the G.C.D of two consecutive numbers. Also, we have used a little bit different approach to show that there must exist at least 3 different primes rather than $\left\{p_{1}, p_{2}, \ldots, p_{r}\right\}$ that satisfy $x^{4}=1$ in $U(n)$.

\section{References}

[1] Meštrović, Romeo. (2012). Euclid's theorem on the infinitude of primes: a historical survey of its proofs (300 B.C.-2012) and another new proof.

[2] W. Dunham, Euler: The Master of Us All, MAA, 1999

[3] Aigner, M. Ziegler, G.M. (1998), Proofs from The Book, Springer, Berlin

[4] Sam Northshield, A One-Line Proof of the Infinitude of Primes, The American Mathematical Monthly, 122(5), p. 466

[5] H. Fürstenberg, On the Infinitude of Primes, Amer. Math. Monthly 62 (1955), 353 